\title{
Desempenho e características de carcaças de cordeiros suplementados com diferentes níveis de resíduo de biodiesel
}

\author{
[Performance and characteristics of lamb carcass supplemented with differents \\ levels of biodiesel residues] \\ B.C. Soares ${ }^{1}$, K.D.S. Souza ${ }^{2}$, J.B. Lourenço Junior ${ }^{3}$, A.G. Maciel e Silva ${ }^{4}$, S.C. Ávila ${ }^{4}$, \\ F. Kuss ${ }^{5}$, S.J.T. Andrade ${ }^{4}$, L.C.B. Raiol ${ }^{6}$, J.C.N Colodo ${ }^{7}$ \\ ${ }^{1}$ Universidade Federal Rural da Amazônia - UFRA - Belém, PA \\ ${ }^{2}$ Zootecnista autônoma \\ ${ }^{3}$ Universidade do Estado do Pará - UEPA/CCNT - Belém, PA \\ ${ }^{4}$ Universidade Federal do Pará - UFPA - Belém, PA \\ ${ }^{5}$ Universidade Tecnológica Federal do Paraná - UTFPR - Curitiba, PR \\ ${ }^{6}$ Instituto de Estudos Superiores da Amazônia - IESAM - Belém, PA \\ ${ }^{7}$ Aluna de pós-graduação - Universidade Federal de Minas Gerais - Belo Horizonte, MG
}

\section{RESUMO}

Estudou-se a suplementação de dietas de cordeiros confinados com resíduo de biodiesel, utilizando-se 25 ovinos, SRD, machos, castrados, com média de peso de $20 \mathrm{~kg}$. O delineamento experimental foi de blocos ao acaso, com cinco repetições por tratamento e esquema de parcelas subdivididas para os dados de biometria. O experimento teve a duração de 70 dias. As dietas eram compostas de $34 \%$ de volumoso e $61 \%$ de concentrado, à base de milho, soja, minerais e 5\% de óleo de dendê ou resíduo de biodiesel, em percentagens crescentes - zero, 25, 50, 75 e $100 \%$. Os animais foram abatidos ao final do experimento para avaliação da carcaça. Foram observados efeitos significativos $(\mathrm{P}<0,05)$ de percentagem de inclusão do resíduo, para as variáveis de desempenho e biometria demonstrados nos efeitos lineares crescentes das equações de regressão, assim como para peso da carcaça quente (PCQ) -, com médias de 14; 15,92; 16,$14 ; 16,42$ e 18,02\% - e peso da carcaça fria (PCF) -, com médias de 13,12; 14,78; 15,06; 15,70 e $17,25 \%$-, para dietas com, respectivamente, $0,25,50,100 \%$ de resíduo de biodiesel de dendê. A utilização de resíduo de biodiesel de dendê na alimentação de cordeiros em crescimento é alternativa para aumentar a densidade energética de suas dietas.

Palavras-chave: ruminantes, subproduto, desempenho e carcaça

\begin{abstract}
Diet supplementation with biodiesel residues of confined lambs, utilizing 25 sheep, without definite breed pattern (WDB), male, castrated, with average weight of $20 \mathrm{~kg}$ was studied. The experimental design was random blocks, with 5 repetitions per treatment and a split plot scheme toward biometrics data. The experiment lasted 70 days. The diets were composed of $34 \%$ of roughage and $61 \%$ of concentrate, based on corn, soy, mineral and 5\% of oil palm kernel or biodiesel residues, in increasing percentages - zero, 25, 50, 75 and 100\%. The animals were slaughtered at the end of the experiment for carcass evaluation. Significant effects $(P<0.05)$ of percentage inclusion of residues were observed for the performance and biometrics variables demonstrated in the crescent linear effect of the regression equations, as well as for the hot carcass weight $(\mathrm{HCW})$ - with an average of $14 ; 15.92 ; 16.14 ; 16.42$ and $18.02 \%$; and the cold carcass weight $(C C W)$ - with an average of 13.12; 14.78; 15.06; 15.70 and $17.25 \%$ for diets, respectively, containing 0,25, 50, 75, 100\% of palm kernel biodiesel residues. The palm kernel biodiesel residue utilization in feeding growing lambs is an alternative to increase the energetic density in their diets.
\end{abstract}

Keywords: ruminants, byproduct, performance and carcass

Recebido em 30 de junho de 2011

Aceito em 10 de setembro de 2012

E-mail: brunocs@veterinario.med.br 


\section{INTRODUÇÃO}

Entre as espécies de ruminantes criados pelo homem para produção de carne, os ovinos e caprinos são os que apresentam o menor intervalo de tempo entre o nascimento e o abate. A carne ovina, em virtude de sua qualidade e valor nutritivo, ocupa posição de destaque na dieta humana, e o desequilíbrio entre a produção e o consumo determina o desenvolvimento das mais variadas estratégias, para permanente abastecimento do mercado consumidor.

Percebe-se o aumento no interesse de intensificar a terminação de cordeiros em confinamento, para rapidez na comercialização e produção de carcaças com melhor rendimento e que atendam às exigências do consumidor moderno, no que se refere ao bem-estar animal, sabor da carne e acabamento de gordura. No entanto, esse tipo de criação requer maior investimento em instalações, alimentação e mão de obra. Uma das formas de viabilizar esse sistema é a utilização de rações formuladas com alimentos alternativos disponíveis regionalmente.

A alimentação dos ruminantes representa o maior custo da atividade pecuária, o que pode representar um entrave para a produtividade, no que concerne ao custo-benefício. Alimentos como milho e farelo de soja são muito utilizados, porém oneram a atividade, uma vez que são produtos, também, representativos na alimentação de não ruminantes e de humanos. Os subprodutos da indústria de transformação de alimentos se tornam alternativa economicamente viável, que podem substituir parcial ou totalmente alimentos volumosos ou concentrados, sem comprometer o desempenho e a eficiência alimentar do animal (Mendonça Júnior, 2009).

O desconhecimento quanto à possibilidade de substituição de alimentos convencionais por subprodutos da agroindústria de menor custo, justifica o desenvolvimento de pesquisas, no sentido de se utilizar alimentos alternativos, com adoção de estratégias de alimentação. Face às considerações descritas, objetivou-se verificar o efeito de diferentes níveis de inclusão do óleo de dendê e resíduo de biodiesel oriundo do dendezeiro (Elaeis guineensis), na dieta de cordeiros confinados, avaliando-se desempenho e características da carcaça.

\section{MATERIAL E MÉTODOS}

O experimento foi conduzido no Instituto Federal de Educação, Ciência e Tecnologia do Pará IFPA/Campus Castanhal, Pará ( $1^{\circ} 17^{\prime} \mathrm{S} /$ $\left.47^{\circ} 55^{\prime} \mathrm{W}\right)$. O tipo climático é Ami, segundo Köppen, com precipitação pluviométrica média de $2.770 \mathrm{~mm} / \mathrm{ano}$, distribuída ao longo do ano. A temperatura média anual é de $26,8^{\circ} \mathrm{C}$, com média de umidade relativa do ar em torno de $85 \%$ (Pacheco e Bastos, 2001; Bastos et al., 2002).

Foram utilizados 25 cordeiros machos, sem raça definida, castrados, com peso vivo médio de $20 \mathrm{~kg}$, distribuídos em delineamento em blocos ao acaso, com cinco tratamentos e cinco repetições por tratamento, sendo cada animal uma repetição. Após sorteio, foram alojados em baias individuais, localizadas em galpão de alvenaria coberto, providas de cochos, com separação para volumoso e concentrado, e bebedouro com capacidade para oito litros de água, em período experimental de 84 dias, sendo quatorze dias de adaptação às dietas e instalações, vermifugação dos animais, e 70 dias para a obtenção de variáveis de desempenho em confinamento.

Os animais receberam água ad libitum e alimento ofertado, que foi regulado, diariamente, para possibilitar $10 \%$ de sobras, dividido em duas refeições, às $7 \mathrm{~h}$ e $17 \mathrm{~h}$. A quantidade ofertada de alimento foi ajustada, de acordo com as sobras do dia anterior, a qual era pesada para avaliação do consumo de matéria seca (CMS). As dietas (Tab. 1) eram compostas de $34 \%$ de feno de Panicum maximum cv. Massai, $61 \%$ de concentrado, à base de milho moído, farelo de soja, minerais, e inclusão de $5 \%$ de óleo de dendê (Tab. 3) ou resíduo de biodiesel de dendê (Tab. 3) e (Tab. 2), misturado manualmente ao concentrado, antes do arraçoamento. O balanceamento da dieta foi feito de acordo com o NRC (National..., 2007), para suprir ganho diário de $0,150 \mathrm{~kg}$. 
Desempenho e características...

Tabela 1. Dietas experimentais - percentagem dos ingredientes (\%)

\begin{tabular}{lcccc}
\hline Tratamento & Feno & Concentrado & Óleo de dendê & Resíduo de biodiesel \\
\hline $100 \%$ dendê & 34,00 & 61,00 & 5,00 & 0,00 \\
$25 \%$ resíduo & 34,00 & 61,00 & 3,75 & 1,25 \\
$50 \%$ resíduo & 34,00 & 61,00 & 2,50 & 2,50 \\
$75 \%$ resíduo & 34,00 & 61,00 & 1,25 & 3,75 \\
$100 \%$ resíduo & 34,00 & 61,00 & 0,00 & 5,00 \\
\hline
\end{tabular}

Tabela 2. Composição bromatológica da dieta, em \% de MS

\begin{tabular}{lccccc}
\multirow{2}{*}{ Composição } & \multicolumn{5}{c}{ Níveis de resíduo na dieta total } \\
\cline { 2 - 5 } & $0 \%$ & $25 \%$ & $50 \%$ & $75 \%$ & $100 \%$ \\
\hline MS & 85,23 & 85,61 & 85,23 & 85,22 & 85,70 \\
MM & 61,40 & 61,12 & 61,58 & 61,57 & 60,67 \\
PB & 12,02 & 11,88 & 11,61 & 11,89 & 11,81 \\
EE & 11,11 & 11,36 & 11,94 & 11,62 & 9,36 \\
FDN & 31,65 & 33,06 & 30,98 & 33,02 & 30,80 \\
FDA & 22,65 & 22,91 & 22,64 & 22,89 & 22,62 \\
Lignina & 3,68 & 3,23 & 3,05 & 3,09 & 3,18 \\
\hline
\end{tabular}

*Ingredientes (milho, farelo de soja, ureia, amireia e minerais).

Tabela 3. Perfil de ácidos graxos da suplementação lipídica em função do nível de substituição do óleo de dendê pelo resíduo do biodiesel

\begin{tabular}{llccccc} 
Ácido graxo & Fórmula & $\begin{array}{c}100 \% \text { Óleo de } \\
\text { dendê }\end{array}$ & $25 \%$ & $50 \%$ & $75 \%$ & $\begin{array}{c}100 \% \text { Resíduo de } \\
\text { biodiesel }\end{array}$ \\
\hline Caprílico & $\mathrm{C} 8: 0$ & - & 0,05 & 0,10 & 0,15 & 0,21 \\
Cáprico & $\mathrm{C} 10: 0$ & - & 0,05 & 0,10 & 0,15 & 0,20 \\
Láurico & $\mathrm{C} 12: 0$ & 0,24 & 0,71 & 1,18 & 1,65 & 2,12 \\
Mirístico & $\mathrm{C} 14: 0$ & 0,78 & 0,86 & 0,94 & 1,02 & 1,11 \\
Palmítico & $\mathrm{C} 16: 0$ & 38,37 & 38,37 & 38,37 & 38,37 & 38,38 \\
Palmitoleico & $\mathrm{C} 16: 1$ & 0,14 & 0,14 & 0,14 & 0,14 & 0,15 \\
Margárico & $\mathrm{C} 17: 0$ & 0,10 & 0,11 & 0,12 & 0,13 & 0,15 \\
Esteárico & $\mathrm{C} 18: 0$ & 5,03 & 4,94 & 4,85 & 4,76 & 4,67 \\
Oleico & $\mathrm{C} 18: 1$ & 43,04 & 41,69 & 40,34 & 38,99 & 37,64 \\
Linoleico & $\mathrm{C} 18: 2$ & 11,36 & 11,78 & 12,21 & 12,63 & 13,06 \\
Linolênico & $\mathrm{C} 18: 3$ & 0,31 & 0,42 & 0,53 & 0,64 & 0,75 \\
Araquídico & $\mathrm{C} 20: 0$ & 0,34 & 0,35 & 0,37 & 0,39 & 0,41 \\
Total & & 99,71 & 99,49 & 99,28 & 99,06 & 98,85 \\
Saturados & & 44,86 & 45,45 & 46,05 & 46,65 & 47,25 \\
Insaturados & & 54,85 & 54,03 & 53,22 & 52,41 & 51,60 \\
Relação S:I & & 0,81 & 0,84 & 0,86 & 0,89 & 0,91 \\
\hline
\end{tabular}

As amostras de oferecido e de sobras foram analisadas para determinação de matéria seca (MS), matéria mineral (MM) e proteína bruta (PB) (Silva e Queiroz, 2002); a fibra em detergente neutro (FDN) e a fibra em detergente ácido (FDA) foram analisadas pelo método sequencial de Van Soest et al. (1991).

No início do experimento e a cada 14 dias foram realizadas pesagens pela manhã, antes da primeira refeição, após 12 h de jejum de sólidos e dieta líquida, sendo avaliado o ganho de peso diário (GPD), calculado com a diferença entre o peso vivo inicial (PVI) e o peso vivo final (PVF), dividido pelo intervalo de tempo entre as coletas (IC) $($ GPD = PVF - PVI)/IC) e a conversão alimentar (CA), por meio da relação entre o consumo de MS (CMS) e o GPD (CA = CMS / GPD).

Previamente à pesagem, procederam-se às medições biométricas, utilizando-se fita métrica flexível para determinação do comprimento corporal (CC), distância entre a articulação 
cérvico-torácica e a base da cauda; altura de cernelha (AC), distância entre uma reta medida da cernelha ao solo; altura de garupa (AG), distância entre uma reta medida da garupa ao solo; perímetro torácico (PT), contorno da circunferência torácica, medida atrás da paleta; largura da garupa (LG), distância entre os trocânteres maiores dos fêmures; largura do peito (LP), distância entre as faces laterais das articulações escápulo-umerais; escore corporal (EC), realizado por meio de exame visual e palpação da região lombar e na inserção da cauda dos animais; comprimento de paleta (Cpa), medida entre articulação rádio-ulna-escapular e extremidade da escápula; comprimento de pernil (Cpe), medida entre o bordo anterior do osso do púbis e o ponto médio dos ossos das articulações do tarso; perímetro de pernil (Ppe), medida da parte média da perna, acima da articulação femorotibial; comprimento de garupa (CG), distância entre a tuberosidade do ílio e o trocânter maior do fêmur, de acordo com Osório et al. (1998). Todas as medidas foram tomadas com os animais dispostos em superfície horizontal e plana e sempre pela mesma pessoa, no intuito de minimizar os erros decorrentes do avaliador.

O abate foi realizado após período de confinamento, com animais em jejum sólido e dieta hídrica de $12 \mathrm{~h}$, e pesados momentos antes do abate (PA). O abate foi realizado através de atordoamento por concussão cerebral e sangria da veia jugular e artéria carótida, segundo o RIISPOA (Brasil, 1997), com coleta e pesagem total do sangue; as carcaças foram penduradas pelos tendões, em ganchos apropriados. Em seguida, foi feita a esfola e abertura da linha mediana ventral, para retirada das vísceras da cavidade torácica (pulmão, coração, traqueia) e ventral (fígado e rins), enquanto os conteúdos do trato digestório, vesícula biliar e bexiga foram obtidos pela diferença de peso cheio e vazio, para obtenção do peso do corpo vazio (PCV).

Após a evisceração e retirada da cabeça e parte distal dos membros, obteve-se o peso da carcaça quente (PCQ), usado para se obter o rendimento da carcaça quente (RCQ), através da equação $(\mathrm{RCQ}=\mathrm{PCQ} / \mathrm{PA} \mathrm{x} 100) . \mathrm{O}$ rendimento biológico (RB) foi obtido a partir da razão entre o PCQ e o peso de corpo vazio (PCV) $(\mathrm{RB}=$ $\mathrm{PCQ} / \mathrm{PCV} \times 100)$. O procedimento seguinte foi a refrigeração da carcaça a $7^{\circ} \mathrm{C}$, em câmara fria, durante 24h. Ao final desse processo, obteve-se o peso da carcaça fria (PCF), perda pelo resfriamento $(\mathrm{PR})(\mathrm{PR}=((\mathrm{PCQ}-\mathrm{PCF}) / \mathrm{PCQ}) \mathrm{x}$ 100) e rendimento da carcaça fria $(\mathrm{RCF})$ ou comercial $(\mathrm{RCF}=\mathrm{PCF} / \mathrm{PA} \times 100)$.

Os animais foram distribuídos em delineamento de blocos ao acaso, com cinco repetições por tratamento, para os dados de ganho de peso e características de carcaça, sendo os blocos em função do peso inicial dos animais. Os dados de biometria foram analisados em esquema de parcelas subdivididas, sendo as parcelas os níveis de resíduo e as subparcelas os tempos de coleta. Para as análises estatísticas foi utilizado o programa NTIA versão 4.2.1 (Embrapa, 1995), onde foram aplicadas análises de regressão e correlação de Pearson. As médias foram comparadas pelo teste Student-Newman-Keuls (SNK), com 5\% de probabilidade.

\section{RESULTADOS E DISCUSSÃO}

Houve efeito linear crescente no PV $(\mathrm{P}<0,05)$, da inclusão do resíduo de biodiesel de dendê (Tab. 4). Observa-se que a cada $1 \%$ de substituição do óleo de dendê pelo resíduo de biodiesel, o PVF aumenta $0,059 \mathrm{~kg}$, segundo a equação $\mathrm{Y}=$ 0,0599x + 28,4 (Tab. 4). Provavelmente, esse efeito está relacionado ao CMS (Tab. 4), que apresentou efeito igual e com melhores resultados na dieta com $100 \%$ de inclusão do resíduo. Palmquist e Mattos (2006) citam que ácidos graxos saturados são menos prejudiciais às fibras que os insaturados, isso devido à toxicidade do ácido graxo aos microrganismos ruminais, que está relacionada à sua natureza anfílica, isto é, aqueles que são solúveis, tanto em solventes orgânicos quanto em água, são mais tóxicos. Tais ácidos incluem os ácidos graxos poli-insaturados de cadeia longa. Esse fato explica os resultados satisfatórios das variáveis estudadas nos animais que estavam em dietas com $100 \%$ de resíduo de biodiesel, pois, na medida em que se aumenta a inclusão do resíduo, também aumenta a proporção de ácidos graxos saturados na dieta (Tab. 3).

A correlação de Pearson apresentou valores positivos para CMS e PV, com coeficiente de determinação de 0,75 . O CMS variou de 0,60 a $0,96 \mathrm{~kg} \mathrm{dia}{ }^{-1}$, em cordeiros que receberam, respectivamente, dietas com 0 e $100 \%$ de resíduo de biodiesel, em substituição ao óleo de dendê, 
cujo CMS da dieta, com o maior valor de inclusão de resíduo, se aproxima do preconizado pelo NRC (2007), para cordeiros de $20 \mathrm{~kg}$, de $1,0 \mathrm{~kg}$ de CMS dia ${ }^{-1}$.

Em resultados semelhantes ao deste trabalho, Hill e West (1991) avaliaram a inclusão de 6\% de óleo de palmáceas na ração e observaram diminuição no ganho de peso de novilhos, devido a efeito negativo no CMS, como constataram Haddad e Younis (2004), com teores crescentes de gordura protegida $(0,2,5$ e $5 \%)$, na ração de cordeiros. Alves et al. (2003) registraram valores inferiores aos do presente trabalho, quando a dieta estava com maiores níveis de inclusão do resíduo de biodiesel, de $0,96 \mathrm{~kg} \mathrm{dia}^{-1}$ (Tab. 4).

Tabela 4. Variáveis de desempenho e biometria in vivo em função do nível de resíduo de biodiesel oriundo do dendê em $5 \%$ da dieta total após 70 dias de confinamento

\begin{tabular}{|c|c|c|c|c|c|c|c|}
\hline \multirow{2}{*}{ Variável } & \multicolumn{5}{|c|}{ Concentrado } & \multirow{2}{*}{ ER } & \multirow{2}{*}{$\mathrm{R}^{2}$} \\
\hline & $0 \%$ & $25 \%$ & $50 \%$ & $75 \%$ & $100 \%$ & & \\
\hline CMS & 0,60 & 0,83 & 0,89 & 0,95 & 0,96 & $\mathrm{Y}=0,0033 \mathrm{x}+0,6802^{*}$ & 0,82 \\
\hline PVF & 28,04 & 30,8 & 31,58 & 31,25 & 35,3 & $Y=0,0599 x+28,4^{*}$ & 0,83 \\
\hline GPD & 0,120 & 0,160 & 0,160 & 0,170 & 0,190 & $Y=0,0007 x+0,109 *$ & 0,84 \\
\hline $\mathrm{CA}$ & 6,18 & 5,80 & 6,62 & 5,90 & 6,00 & ns & - \\
\hline EC & 2,36 & 2,58 & 2,56 & 2,58 & 2,88 & $Y=0,004 x+2,38^{*}$ & 0,78 \\
\hline $\mathrm{CC}$ & 60,66 & 60,98 & 63,06 & 62,24 & 63,20 & $Y=0,025 x+60,74 *$ & 0,75 \\
\hline PT & 66,30 & 68,18 & 69,21 & 68,32 & 69,40 & $Y=0,0254 x+67,044^{*}$ & 0,63 \\
\hline Cpa & 20,04 & 20,50 & 20,83 & 20,08 & 21,22 & ns & - \\
\hline LP & 18,64 & 19,02 & 19,63 & 19,84 & 19,86 & $Y=0,013 x+18,75^{*}$ & 0,88 \\
\hline LG & 14,52 & 15,36 & 14,81 & 14,78 & 14,82 & ns & - \\
\hline CG & 20,20 & 19,30 & 20,33 & 20,28 & 20,30 & ns & - \\
\hline Cpe & 22,90 & 22,82 & 23,30 & 23,24 & 23,16 & ns & - \\
\hline Ppe & 38,98 & 41,24 & 42,86 & 40,64 & 42,12 & ns & - \\
\hline $\mathrm{AC}$ & 60,16 & 58,68 & 60,54 & 61,70 & 61,62 & $\mathrm{~ns}$ & - \\
\hline AG & 62,50 & 64,52 & 62,76 & 63,14 & 64,00 & ns & - \\
\hline
\end{tabular}

CMS = Consumo de matéria seca $; \mathrm{PV}=$ Peso vivo $; \mathrm{GPD}=$ Ganho de peso diário; $\mathrm{CA}=$ Conversão Alimentar; $\mathrm{EC}=$ Escore corporal $\mathrm{CC}=$ Comprimento corporal; $\mathrm{PT}=$ Perímetro torácico; $\mathrm{Cpa}=$ Comprimento de paleta; $\mathrm{LP}=\mathrm{Largura}$ de peito; $\mathrm{LG}=$ Largura de garupa; $\mathrm{CG}=$ Comprimento de garupa; $\mathrm{Cpe}=$ Comprimento de pernil; Ppe $=$ Perímetro de pernil; $\mathrm{AC}=$ Altura de cernelha; $\mathrm{AG}=$ Altura de garupa; $\mathrm{ER}=$ Equação de regressão; $\mathrm{R}^{2}=$ Coeficiente de determinação. *significativo $(\mathrm{P}<0,05) ;$ ns $=$ não significativo.

No que concerne às correlações entre as características mensuradas, foram constatados valores positivos entre o CMS e as variáveis EC, CC, PT, Cpa, CG, Ppe, AC e AG, com coeficientes de correlação, respectivamente, de 0,$86 ; 0,87 ; 0,86 ; 0,83 ; 0,71 ; 0,81 ; 0,70$ e 0,77 $(\mathrm{P}<0,05)$, o que aponta melhor desempenho para o tratamento com maior CMS.

O GPD com $100 \%$ de resíduo foi de $0,190 \mathrm{~kg}$ animal $^{-1} \mathrm{dia}^{-1}$ (Tab. 4). Isso indica efeito linear crescente $(\mathrm{P}<0,05)$, com consequente efeito similar do PV, o que demonstra que, quanto maior o nível de inclusão de resíduo, maior é o desempenho animal. Esses valores estão próximos ao proposto pelo NRC (2007), de $0,200 \mathrm{~kg}$. Ao contrário deste trabalho, Rizzi et al.
(2002) não verificaram alteração no GPD com uso de fontes e teores de gordura na ração; da mesma forma, Cunha et al. (2008), com níveis crescentes de caroço de algodão integral, não registraram aumento no GPD de cordeiros. Yamamoto et al. (2005) observaram GPD superiores aos relatados neste estudo, com uso de dietas de diferentes óleos vegetais, enquanto Dutta et al. (2008) comprovaram que o melhor ganho de peso foi na dieta com 5\% de óleo de dendê, e Garcia et al. (2003) observaram resultado semelhante, com efeito linear crescente, ao avaliarem níveis crescentes de energia na ração de cordeiros.

Houve efeito linear para o comprimento corporal (CC) $(\mathrm{P}<0,05)$ (Tab. 4), em que as medidas 
crescem $0,025 \mathrm{~cm}$, a cada $1 \%$ de inclusão do resíduo, segundo a equação $\mathrm{Y}=0,025 \mathrm{x}+60,74$ (Tab. 4). Apesar das dietas estudadas terem valores próximos, a com $100 \%$ de substituição do óleo de dendê pelo resíduo de biodiesel apresentou os melhores resultados.

As medidas biométricas de PT e LP foram semelhantes (Tab. 4). Os valores de LP (Tab. 3) variaram entre 18,64 e 19,86 e foram inferiores aos observados por Moreno et al. (2010b), de $25,23 \mathrm{~cm}$, e Moreno et al. (2010a), entre 25,10 e $25,98 \mathrm{~cm}$, cujas diferenças podem ser atribuídas ao tipo de animal usado que, neste trabalho, eram SRD. Para PT, as medidas variaram entre 66,30 e $69,40 \mathrm{~cm}$, valores que demonstram que a dieta com $100 \%$ de resíduo propicia melhor desempenho.

O PV apresentou correlação positiva $(\mathrm{P}<0,05)$ para as medidas biométricas, exceto LP e LG, porém, com melhor coeficiente com o PT $(0,92)$, semelhante ao encontrado na literatura (Santana et al., 2001; Pinheiro e Jorge, 2010), que indica que a medida que melhor explica o peso corporal é o perímetro torácico. O PT apresentou elevada correlação com Cpa e Ppe, respectivamente, de 0,92 e 0,97 , consideradas relevantes, pois constituem cortes nobres para o mercado consumidor.

Com relação às variáveis de carcaça, não houve interação entre dietas e tempo de coleta, nas variáveis avaliadas. Os pesos de carcaça quente (PCQ) e fria (PCF) (Tab. 5) apresentaram efeito linear crescente $(\mathrm{P}<0,05)$, em função do nível de resíduo nas dietas, assim como as varáveis de desempenho e medidas biométricas. Os PCQ aumentam $0,0342 \mathrm{~kg}$ (Tab. 5), a cada $1 \%$ de inclusão do resíduo de biodiesel de dendê, que determina rendimentos (RCQ) de 49,62 a $51,01 \%$, respectivamente, nas dietas de 0 a $100 \%$ de resíduo, em relação aos pesos vivos ao abate. Esses rendimentos são superiores aos obtidos por Alves et al. (2003); Zundt et al. (2006) e Lombardi et al. (2010). O PCQ apresentou alta correlação positiva com as variáveis PCF, GPD e CMS, respectivamente, com coeficientes de correlação de 0,99; 0,99; e 0,92. O PCF, também, apresentou alta correlação positiva com GPD $(0,98)$.

Tabela 5. Variável de carcaça, em função do nível de resíduo, em 5\% da dieta total

\begin{tabular}{|c|c|c|c|c|c|c|c|}
\hline \multirow{2}{*}{ Variável } & \multicolumn{5}{|c|}{ Nível de resíduo na dieta } & \multirow[b]{2}{*}{ ER } & \multirow[b]{2}{*}{$\mathrm{R}^{2}$} \\
\hline & $0 \%$ & $25 \%$ & $50 \%$ & $75 \%$ & $100 \%$ & & \\
\hline PCQ & 14 & 15,92 & 16,14 & 16,42 & 18,02 & $Y=0,0342 x+14,392 *$ & 0,88 \\
\hline PCF & 13,12 & 14,78 & 15,06 & 15,70 & 17,25 & $Y=0,0368 x+13,345^{*}$ & 0,93 \\
\hline RCQ & 49,62 & 51,47 & 51,19 & 52,96 & 51,01 & $Y=0,0006 x^{2}+0,0804 x+49,604 *$ & 0,706 \\
\hline $\mathrm{RCF}$ & 45,03 & 47,69 & 45,91 & 50,60 & 47,70 & Ns & - \\
\hline $\mathrm{RB}$ & 59,54 & 59,54 & 59,82 & 59,73 & 58,64 & $Y=-0,0003 x^{2}+0,0227 x+59,412 *$ & 0,814 \\
\hline PR & 6,28 & 7,38 & 6,77 & 4,37 & 4,54 & Ns & - \\
\hline
\end{tabular}

$\mathrm{PCQ}=$ Peso da carcaça quente; $\mathrm{PCF}=$ Peso da carcaça fria; $\mathrm{RCQ}=$ Rendimento da carcaça quente; $\mathrm{RCF}=$ Rendimento da carcaça fria; RB = Rendimento biológico; PR = Perda por resfriamento; ER = Equação de regressão; $\mathrm{R}^{2}=$ Coeficiente de determinação; $*(\mathrm{P}<0,05) ; \mathrm{ns}=$ não significativo.

Observa-se influência do CMS e PV dos animais, com uso de resíduo de biodiesel na alimentação de cordeiros, que propiciou efeito linear crescente no PCQ e, consequentemente, no RCQ (Tab. 5) e RB (Tab. 5), o que demonstrou melhor rendimento à medida que se substituiu o óleo pelo resíduo. O RCQ apresentou alta correlação positiva com RCF $(0,92) \quad(\mathrm{P}<0,05)$, resultado semelhante ao de Piola Jr. et al. (2009), em que as maiores correlações foram entre PCF e PCQ, e RCQ e RCF.

A equação de regressão apresentou efeito quadrático para a variável RCQ, com rendimentos entre 49,62 e $51,01 \%$ (Tab. 5), respectivamente, em dietas com 0 a $100 \%$ de inclusão do resíduo. Efeito semelhante se manifestou para o RB, com valores próximos 
entre os tratamentos. Por derivação das equações, o nível de inclusão do resíduo de biodiesel de dendê foi de $67 \%$ e $37,83 \%$, respectivamente, para os melhores resultados de RCQ $(52,30 \%)$ e RB (59,84\%), superiores ao relatado por Rocha et al. (2004); Urano et al. (2006), Dantas Filho et al. (2008); e semelhante ao de Garcia et al. (2003), de 52,76\% RCQ.

Entre os fatores que influenciam no rendimento da carcaça de ruminantes, destaca-se a dieta (Siqueira e Fernandes, 1999). Assim, o efeito quadrático na equação de regressão, para RCQ e $\mathrm{RB}$, indica que, apesar da redução de rendimento nas dietas com $100 \%$ de óleo de dendê ou resíduo, os resultados são satisfatórios, quando comparados aos da literatura.

\section{CONCLUSÕES}

A utilização de resíduo de biodiesel de dendê em substituição ao óleo de dendê na alimentação de cordeiros promove efeito crescente no consumo de matéria seca e ganho de peso, o que melhora as características de carcaça. Esses coprodutos da agroindústria apresentam-se como alternativa para aumentar a densidade energética de dietas para ovinos em crescimento.

\section{AGRADECIMENTOS}

Ao IFPA/Campus Castanhal, PA, a Faculdade de Medicina Veterinária/UFPA/Campus Castanhal, PA, ao Laboratório de Nutrição Animal da UFMG, MG, ao PROCAD - NF N ${ }^{\circ}$ 08/2008 CAPES e a FAPESPA.

\section{REFERÊNCIAS}

ALVES, K.S.; CARVALHO, F.F.R.; FERREIRA, M.A. Níveis de energia em dietas para ovinos Santa Inês: características de carcaça e constituintes corporais. Rev. Bras. Zootec., v.32, p.1927-1936, 2003.

BASTOS, T.X.; PACHECO, N.A.; NECHET, D.N. et al. Aspectos climáticos de Belém, nos últimos cem anos. Belém: Embrapa Amazônia Oriental, 2002.31p.

BRASIL. Ministério da Agricultura Pecuária e Abastecimento - MAPA. Regulamento da Inspeção Industrial e Sanitária de Produtos de Origem Animal. Brasília: 1997. 154p.
CUNHA, M.G.G.; CARVALHO, F.F.R.; VÉRAS, A.S.C. et al. Desempenho e digestibilidade aparente em ovinos confinados alimentados com dietas contendo níveis crescentes de caroço de algodão integral. Rev. Bras. Zootec., v.37, p.1103-1111, 2008.

DANTAS, A.F.; PEREIRA FILHO, J.M.; SILVA, A.M.A. et al. Características da carcaça de ovinos Santa Inês terminados em pastejo e submetidos a diferentes níveis de suplementação. Cienc. Agrotec., v.32, p.1280-1286, 2008.

DUTTA, T.K.; AGNIHOTRI, M.K.; RAOC, S.B.N. Effect of supplemental palm oil on nutrient utilization, feeding economics and carcass characteristics in post-weaned Muzafarnagari lambs under feedlot condition. Small Rum. Res., v.78, p.66-73, 2008.

EMBRAPA, Empresa brasileira de pesquisa agropecuária. NTIA, versão 4.2.1. Campinas-SP 1995.

GARCIA, C.A.; COSTA, C.; MONTEIRO, A.L.G. et al. Níveis de energia no desempenho e características da carcaça de cordeiros alimentados em "creep feeding". Rev. Bras. Zootec., v.32, p.1371-1379, 2003.

HADDAD, S.G.; YOUNIS, H.M. The effect of adding ruminally protected fat in fattening diets of nutrient intake, digestibility and growth performance of Awassi lambs. Anim. Feed Sci. Tech., v.113, p.61-69, 2004.

HILL, G.M.; WEST, J.W. Rumen protected fat in kline barley or corn diets for beef cattle: Digestibility, physiological and feedlot responses. J. Anim. Sci., Champaign, v. 69, p.376-38, 1991.

LOMBARDI L.; JOBIM, C.C.; BUMBIERIS JÚNIOR, V.H. et al. Características da carcaça de cordeiros terminados em confinamento recebendo silagem de grãos de milho puro ou com adição de girassol ou ureia. Acta Sci. Anim. Sci., v.32, p.263-269, 2010.

MENDONÇA JUNIOR, A.F. Características de carcaça, componentes não carcaça e qualidade da carne de ovinos alimentados com dietas a base de palma forrageira (Opuntia fícus indica) $e$ diferentes fontes de fibra. 2009. 28f. Dissertação (Mestrado em Zootecnia) Universidade Federal Rural de Pernambuco, PE.

MORENO, G.M.B.; SILVA SOBRINHO, A.G.; LEÃO, A.G. et al. Características morfológicas 
"in vivo" e da carcaça de cordeiros terminados em confinamento e suas correlações. Rev. Bras. Saúde Prod. Anim., v.11, p.888-902, 2010a.

MORENO, G.M.B.; SILVA SOBRINHO, A.G.; ROSSI, R.C. et al. Desempenho e rendimentos de carcaça de cordeiros Ile de France desmamados com diferentes idades. Rev. Bras. Saúde Prod. Anim., v.11, p.1105-1116, 2010b.

NATIONAL research council (NRC). Nutrient Requirements of small ruminants: sheep, goats, cervids and new world camelids. 6th ed. Washington: The National Academy Press: 2007, $384 \mathrm{p}$.

OSÓRIO, J.C.S.; OSÓRIO, M.T.M.; JARDIM, P.O. et al. Métodos para avaliação da produção de carne ovina: in vivo, na carcaça e na carne. Pelotas: Editora Universitária/UFPEL, 1998. p.107.

PACHECO, N.A.; BASTOS, T.X. Caracterização Climática do Município de Tomé-Açu, PA. Belém: Embrapa Amazônia Oriental, 2001. 18p. (Embrapa Amazônia Oriental. Documentos, 87).

PALMQUIST, D.L.; MATTOS, W.R.S. Metabolismo de lipídeos. In: BERCHIELLI, T.T. et al. Nutrição de ruminantes. Jaboticabal: FUNEP, 2006. Cap.10, p.287-310.

PINHEIRO, R.S.B.; JORGE, A.M. Medidas biométricas obtidas in vivo e na carcaça de ovelhas de descarte em diferentes estágios fisiológicos. Rev. Bras. Zootec., v.39, p.4404545, 2010 .

PIOLA JÚNIOR, W; RIBEIRO, E.L.A.; MIZUBUTI, I.Y. et al. Ganho de peso e características da carcaça de cordeiros recebendo diferentes níveis de energia na ração. 1 Semina: Rev. Bras. Cienc. Agrar., v.30, p.935-944, 2009.

RIZZI, L.; SIMIOLI, M.; SARDI, L.; MONETTI, P.G. Carcass quality, meat chemical and fatty and fatty acid composition of lambs fed diets containing extruded soybeans and sunflower seeds. Anim. Feed Sci. Tech., v.97, p.103-114, 2002.

ROCHA, M.G.; MONTAGNER, D.B.; SANTOS, D.T. et al. Parâmetros produtivos de uma pastagem temperada submetida a alternativas de utilização. Rev. Bras. Zootec., v.33, p.1386-1395, 2004.

SANTANA, A.F.; COSTA, G.B.; FONSECA, L.S. Correlações entre peso e medidas corporais em ovinos jovens da raça Santa Inês. Rev. Bras. Saúde Prod. Anim., v.1, p.74-77, 2001.

SILVA, D.J.; QUEIROZ, A.C. Análises de alimentos: Métodos químicos e biológicos. 3. ed. Viçosa: UFV, 2002.

SIQUEIRA, E.R.; FERNANDES, S. Peso, rendimentos e perdas da carcaça de cordeiros Corriedale e mestiços Ile de France X Corriedale, terminados em confinamento. Cienc. Rural, v.29, p.143-148, 1999.

URANO, F.S.; PIRES, A.V.; SUSIN, I. et al. Desempenho e características da carcaça de cordeiros confinados alimentados com grão de soja. Pesq. Agropec. Bras., v.41, p.1525-1530, 2006.

VAN SOEST, P.J.; ROBERTSON, J.B.; LEWIS, B.A. Methods for dietary fiber, neutral detergent fiber and no starch polysaccharides in relation to animal nutrition. J. Dairy Sci., v.74, p.35833597.,1991.

YAMAMOTO, S.M.; MACEDO, F.A.F.; ZUNDT, M. et al. Fontes de Óleo Vegetal na Dieta de Cordeiros em Confinamento. Rev. Bras. Zootec., v.34, p.703-710, 2005.

ZUNDT, M.; MACEDO, F.A.F.; ASTOLPHI, J.L.L. et al. Desempenho e características de carcaça de cordeiros Santa Inês confinados filhos de ovelhas submetidas à suplementação alimentar durante a gestação. Rev. Bras. Zootec., v.35, p.928-935, 2006. 